\title{
Introduction to emerging vector-borne disease and cities workshop proceedings
}

\author{
Karmali $\mathrm{M}^{1 *}$, Weinstock $\mathrm{D}^{1}$
}

\author{
Affiliation \\ ${ }^{1}$ Institute for Health and Social Policy, McGill University, Montréal, QC
}

*Correspondence: mkarmali@rogers.com

Suggested citation: Karmali M, Weinstock D. Introduction to emerging vector-borne disease and cities workshop proceedings. Can Comm Dis Rep 2016;42:197. https://doi.org/10.14745/ccdr.v42i10a01

\section{Introduction}

Cities throughout the world are experiencing spectacular growth in size and in population and have become focal points for globalization, including the rapid movement of people, foods, goods and infectious diseases across borders. Of particular concern are the persistent and expanding mosquito-borne epidemics caused by dengue, chikungunya and Zika viruses. These outbreaks may be further exacerbated by climate change and poor social conditions, especially in low- and middle-income countries. In Canada, the main vector-borne diseases (VBDs) are the tick-borne Lyme disease and the mosquito-borne West Nile virus infection which are also becoming urbanized and whose incidence is also influenced by climate warming.

In many countries, epidemics of chronic diseases such as obesity, type 2 diabetes, cardiovascular and respiratory diseases have been undermining the health of urban populations over the past few decades. To address this, contemporary urban planning in Canada and other developed countries has emphasized reducing urban and suburban sprawl and promoting a "greener", "bluer", urban landscape that contains fewer automobiles and cleaner air. The goal is to enable people to walk and bicycle more and drive less, minimizing pollution and preserving the periurban natural environment.

However, we do not know to what extent the continued expansion of the built (i.e., the urban or suburban) environment and efforts to promote healthier living, may unintentially influence the survival and replication of mosquitos and ticks and people's exposure to them. This could occur due to a greener, bluer landscape, pools of stagnant water in the urban environment and the "heat island" effect whereby temperatures inside cities are much higher than surrounding areas. These effects may be further exacerbated by climate change.

Furthermore, increased urban population densities and the entry of infected travelers and migrants into cities increase the risks of mosquito-borne virus transmission.

In order to explore this issue, an invitational multidisciplinary and multi-sectorial workshop, hosted by the Institute of Health and Social Policy, McGill University was held on April 25-26, 2016. The goal of the workshop was to explore emerging VBDs and the effects of climate change, urban planning strategies for healthier living and mitigation strategies for emerging VBDs. Speakers and participants included experts and policy makers from public health, infectious diseases, epidemiology, health and social policy, urban planning, economics, ecology, sociology, engineering and climate science. Over the two days, there were formal presentations by experts and inter-disciplinary dialogue with a view to identifying mitigating strategies and research gaps pertaining to emerging challenges of VBDs in Canadian cities in the context of climate change and healthy urban living.

In this issue of the journal, we present abstracts of the papers presented followed by a summary of the workshop presentations, discussions and next steps including research questions to advance this field of inquiry.

Emerging VBDs and climate change are real threats to the future well-being of Canadians including those residing in cities. This workshop provided an entrée and impetus for experts and policymakers from different sectors and disciplines to consider the multifaceted approaches that will be needed to address this challenge.

\section{Conflict of interest}

None. 